This is the peer reviewed version of the following article: He Xuezhong and Shi Lei 2012, 'Disagreement in a multiasset market', Blackwell, vol. 12, no. 3, pp. 357-373. which has been published in final form at http://dx.doi.org/10.1111/j.1468-2443.2012.01153.x This article may be used for non-commercial purposes in accordance With Wiley Terms and Conditions for self-archiving' 


\title{
DISAGREEMENT IN A MULTI-ASSET MARKET
}

\author{
XUE-ZHONG HE AND LEI SHI \\ Finance Discipline Group \\ UTS Business School \\ University of Technology, Sydney \\ PO Box 123 Broadway \\ NSW 2007, Australia
}

We would like to thank Kristoffer Glover, Paul Kofman, and Gordon Menzies for helpful comments and conference participants at 2009 KIER-TMU International Workshop on Financial Engineering (Tokyo, Japan), 2009 PhD Conference in Economics and Business (Perth, Australia), and 2010 Asian Finance Association Conference (Hong Kong). In particular we would like to thank the editor, Michael Lemmon, and a referee for their helpful comments and valuable suggestions which have significantly improved the paper. The usual caveat applies. Financial supports from the Australian Research Council (ARC) under Discovery Grant (DP0773776) is gratefully acknowledged.

Corresponding author: Xue-Zhong (Tony) He, UTS Business School, University of Technology, Sydney, PO Box 123 Broadway, NSW 2007, Australia. Email: Tony.He1@uts.edu.au. Ph: (61 2) 9514 7726. Fax: (61 2) 95147722. 


\title{
Disagreement in a Multi-Asset Market
}

\begin{abstract}
This paper provides a simple framework to study the effect of disagreement on a multiasset market equilibrium by considering two agents who disagree about expected returns, variances and correlation of returns of two risky assets. When agents' subjective beliefs are characterized by mean preserving spreads of a benchmark homogeneous belief, we show that the effect of the disagreement does not cancel out in general and the effect in a multi-asset market can be very different from a single asset market. In particular, the market risk premium can increase and the risk-free rate can decrease significantly even when the market is optimistic and confident.
\end{abstract}

JEL Classification: G12, D84.

Keywords: equilibrium asset pricing; heterogeneous beliefs; disagreement; optimism/pessimism; confident/doubt; market risk premium; risk-free rate. 


\section{INTRODUCTION}

There is a growing literature on the behaviour of asset prices under heterogeneous beliefs. Some focus on the aggregation of beliefs and the structure of asset prices under heterogeneous beliefs] others on resolving the equity premium and the risk-free rate puzzles (posed by Mehra and Prescott (1985) and Weil (1989)), explaining the excess volatility puzzle, term structure of interest rates, trading volume of stocks and options, market over(under)-reaction and momentum, and the survival of the irrational agents $\mathrm{s}^{2}$. Most of this literature views the aggregate market as a single risky asset. It is found that heterogeneous beliefs can lead to pessimism and doubt at the market aggregate level, which in turn help to explain high risk premium and low risk-free rate observed in historical data. In this paper, we show that the impact of heterogeneous beliefs in a market with multi-risky assets can be very different from a market with a single risky asset. In particular, there can be a significant increase in the market risk premium and a decrease in the risk-free rate even when the market is over-optimistic and over-confident.

When agents agree to disagree, they may differ in their (subjective) beliefs about the distribution of asset returns. When the subjective beliefs are independent across agents, the heterogeneity in beliefs should cancel out in aggregation and hence have no impact on the market equilibrium. This argument is intuitively appealing. However, in a simple mean-variance framework, Fama and French (2007) show that the effect of heterogeneity in beliefs only cancels out if the mis-informed investors on aggregate hold the market portfolio. Otherwise, there can be a large pricing effect. This is also the case when investors' demands are nonlinear functions of their beliefs, as shown in Yan (2010). Duchin and Levy (2010) also show that agents' demand functions are nonlinear in beliefs of variances of asset returns and the disagreement about the variance can provide a potential explanation for the excess volatility puzzle. When agents on average have the correct belief about the expected growth rate of aggregate endowment, Jouini and Napp (2011) show the existence of waves of pessimism and optimism which in turn lead to countercyclical market prices of risk and a procyclical risk-free rate observed in financial markets.

When the effect of heterogeneity in beliefs does not cancel out, it can have a significant impact on the equity risk premium and the risk-free rate, and the impact on a

\footnotetext{
${ }^{1}$ See, for example, Lintner (1969), Rubinstein (1974, 1975), Detemple and Murthy (1994), Jouini and Napp (2006, 2007) and Chiarella, Dieci and He (2010, 2011).

${ }^{2}$ See, for example, Zapatero (1998), Basak (2000, 2005), Buraschi and Jiltsov (2006), Blume and Easley (2006) and Kogan, Ross, Wang and Westerfield (2006), Li (2007), Hong and Stein (2007), David (2008), Dumas, Kurshev and Uppal (2009), Berraday (2009), Cao and Ou-Yang (2009), and Xiong and Yan (2010).
} 
multi-asset market can be very different from a single-asset market. When the aggregate market is treated as a single risky asset, Abel (2002) shows that there is an increase in the risky premium and a reduction in the risk-free rate if the representative investor is pessimistic and doubtful about the future returns of the market Jouini and Napp (2006) further show that a positive correlation between risk tolerance and pessimism (doubt) ${ }^{4}$ can generate pessimism (doubt) at the aggregate level, leading to higher equity risk premium and lower risk-free rate in compare with a benchmark economy with a homogeneous belief.

In a market of many risky assets, agents may disagree about the joint probability distributions of the risky assets, which can change the market portfolio significantly. Consequently, a multi-asset market can have very different characteristics in compare with the market of single risky asset. However, when the number of risky assets increases in the market, so does the dimension of heterogeneity in beliefs, which complicates the analysis. This paper contributes to the literature by considering a market with multi-risky assets and a risk-free asset. Agents are heterogeneous in their beliefs of the expected returns, variances, and covariance of asset returns, including the heterogeneity in beliefs about the correlation between asset returns (which does not exist in a market with a single risky asset). Within a mean-variance framework, a CAPM can be derived under a consensus belief constructed from the heterogeneous beliefs. To examine the effect of heterogeneity on the market equilibrium, in particular, on the risk premium and the risk-free rate explicitly, we consider a market with two risky assets and two agents whose beliefs are mean preserving spreads (MPS) of the benchmark homogeneous and objective belief. With the MPS, agents perceive the objective expected returns, variances and correlation on average. However, when different aspects of the disagreement are correlated, we show that the effect of the disagreement does not cancel out. Different from the market with single risky asset, there is a spill-over effect, meaning that the dispersion in beliefs about one asset can affect the consensus belief of both risky assets. Furthermore, when the heterogeneity affects the composition of the market portfolio, the multiple risky assets cannot be considered as a whole through the market portfolio. Therefore, the intuitions from the market with a single risky asset may not carry over to a market with multiple risky assets. In particular, a positive correlation between risk tolerance and pessimism/doubt is no longer necessary to generate a high market risk premium and a low risk-free rate. In some cases,

\footnotetext{
${ }^{3}$ When the distributions of returns are normal, being pessimistic means perceiving a lower expected return than the one under the objective belief, and being doubtful means perceiving a larger standard deviation of returns than the one under the objective belief.

${ }^{4}$ Meaning that more risk tolerant agents perceive low expected returns (are more doubtful).
} 
a positive correlation between the optimism and the belief about the correlation between asset returns dramatically increases the market risk premium and decreases the risk-free rate.

The paper is structured as follows. In Section III market equilibrium is characterized by a CAPM under the consensus belief. Section III examines the impact of heterogeneity on the consensus belief, and Section $[\mathrm{IV}$ analyzes the effect on the market risk premium and the risk-free rate. The paper concludes in Section $\mathrm{V}$

\section{Market Equilibrium Under Heterogeneous Beliefs}

In this section, we first set up a stylized two-date economy with heterogeneous beliefs and multiple risky assets. Market equilibrium is then characterized by CAPM under the consensus belief. To examine the effect of the heterogeneity in beliefs and risk tolerances, we also introduce a benchmark economy with a homogenous belief.

A. An Economy under Heterogeneous Beliefs. Consider a two-date economy consisting of $K$ risky assets, indexed by $k=1,, 2, \cdots, K$, and a riskless asset. The return of asset $k$ is denoted by $r_{k}$, return of the riskless asset is denoted by $r_{f}$. The returns of the risky assets are multivariate normal. There are $N$ agents, indexed by $i=$ $1,2, \cdots, N$, who have heterogeneous beliefs about the expected returns and the covariance matrix. Agent $i$ 's subjective beliefs about the expected returns and the covariance matrix are given by a vector $\boldsymbol{\mu}_{i}=\left(\mu_{i, 1}, \mu_{i, 2}, \cdots, \mu_{i, K}\right)^{T}$ and $\boldsymbol{\Sigma}_{i}=\left(\rho_{i, j k} \sigma_{i, j} \sigma_{i, k}\right)_{K \times K}$, respectively, where $\mu_{i, k}=\mathbb{E}_{i}\left[r_{k}\right], \sigma_{i, k}^{2}=\operatorname{Var}_{i}\left[r_{k}\right]$ and $\rho_{i, j k}=\operatorname{Correl}_{i}\left[r_{j}, r_{k}\right]$. We use $\mathcal{B}_{i}:=\left(\boldsymbol{\mu}_{i}, \boldsymbol{\Sigma}_{i}\right)$ to denote the subjective belief of agent $i$.

Let $W_{i, 0}$ be the initial wealth of agent $i, \pi_{i}=\left(\pi_{i, 1}, \pi_{i, 2}, \cdots, \pi_{i, K}\right)^{T}$ be the vector of dollar amount agent $i$ invested in the risky assets, $\mathbf{r}=\left(r_{1}, r_{2}, \cdots, r_{K}\right)^{T}$ and $\mathbf{1}=(1,1, \cdots, 1)^{T}$. Then the terminal wealth of agent $i$ is given by $W_{i}=W_{i, 0}(1+$ $\left.r_{f}\right)+\boldsymbol{\pi}_{i}^{T}\left(\mathbf{r}-r_{f} \mathbf{1}\right)$. Assume that agent $i$ maximizes an expected constant absolute risk aversion (CARA) utility function of the terminal wealth under his subjective belief, that is, $\mathbb{E}_{i}\left[U_{i}\left(W_{i}\right)\right]=\mathbb{E}_{i}\left[-\exp \left\{-W_{i} / \tau_{i}\right\}\right]$, where $\tau_{i}$ is the risk-tolerance of agent $i$. Then the optimal portfolio of agent $i$ is given by $\boldsymbol{\pi}_{i}^{*}=\tau_{i} \boldsymbol{\Sigma}_{i}^{-1}\left(\boldsymbol{\mu}_{i}-r_{f} \mathbf{1}\right)$.

B. Consensus Belief and Market Equilibrium. To characterize market equilibrium, we construct a consensus belief (as in Lintner (1969), Rubinstein (1974,1975) and Chiarella, Dieci and He (2011)) to describe market equilibrium. A belief $\mathcal{B}_{a}=\left(\boldsymbol{\mu}_{a}, \boldsymbol{\Sigma}_{a}\right)$ is called a market consensus belief if the equilibrium prices of the risky assets and the risk-free rate under the heterogeneous beliefs $\mathcal{B}_{i}(i=1,2, \cdots, N)$ are also those under the homogeneous belief $\mathcal{B}_{a}$. Intuitively, the consensus belief reflects how the market as a whole perceives the expected returns and the covariance matrix of returns when market is in equilibrium. 
We normalize the average initial wealth of the market to one, that is $\frac{1}{N} W_{m, 0}=$ $\frac{1}{N} \sum_{i=1}^{N} W_{i, 0}=1$. Then the market clearing condition for risky assets is given by $\frac{1}{N} \sum_{i=1}^{N} \boldsymbol{\pi}_{i}^{*}=\boldsymbol{\pi}_{m}$, where $\boldsymbol{\pi}_{m}$ denotes the market portfolio weights (proportions of initial wealth of the market invested in risky assets) $)^{5}$. Applying Proposition 1 and Corollary 2 in Chiarella, Dieci and He (2010), the consensus belief $\mathcal{B}_{a}$ of the market equilibrium is given by

$$
\boldsymbol{\Sigma}_{a}^{-1}=\frac{1}{N}\left[\sum_{i=1}^{N} \frac{\tau_{i}}{\tau_{a}} \boldsymbol{\Sigma}_{i}^{-1}\right], \boldsymbol{\mu}_{a}=\frac{1}{N}\left[\sum_{i=1}^{N} \frac{\tau_{i}}{\tau_{a}} \boldsymbol{\Sigma}_{a} \boldsymbol{\Sigma}_{i}^{-1} \boldsymbol{\mu}_{i}\right],
$$

where $\tau_{a}:=\frac{1}{N} \sum_{i=1}^{N} \tau_{i}$ is the average risk tolerance of the agents. The consensus belief, in terms of the precision matrix (inverse of the covariance) is a risk tolerance wighted average of agents' subjective beliefs, and in terms of expected returns is a risk tolerance/precision matrix wighted average of the subjective beliefs. Under the consensus belief, the market portfolio in equilibrium is given by

$$
\boldsymbol{\pi}_{m}=\tau_{a} \Sigma_{a}^{-1}\left(\boldsymbol{\mu}_{a}-r_{f} \mathbf{1}\right)
$$

Furthermore, the CAPM

$$
\mathbb{E}_{a}(\mathbf{r})-r_{f} \mathbf{1}=\boldsymbol{\beta}\left[\mathbb{E}_{a}\left(\mathbf{r}_{m}\right)-r_{f}\right]
$$

holds under the consensus belief with $\boldsymbol{\beta}=\boldsymbol{\Sigma}_{a} \boldsymbol{\pi}_{m} /\left(\boldsymbol{\pi}_{m}^{T} \boldsymbol{\Sigma}_{a} \boldsymbol{\pi}_{m}\right)$.

The market risk premium and the risk-free rate are endogenously determined by the consensus belief. In equilibrium, the riskless asset is in zero net supply, which implies that the portfolio weights of the market portfolio must sum up to one, $\boldsymbol{\pi}_{m}^{T} \mathbf{1}=1$. Hence, the equilibrium risk-free rate can be determined by

$$
r_{f}=\left(\mathbf{1}^{T} \boldsymbol{\Sigma}_{a}^{-1} \boldsymbol{\mu}_{a}-1 / \tau_{a}\right) / \mathbf{1}^{T} \boldsymbol{\Sigma}_{a}^{-1} \mathbf{1} .
$$

It follows from equation (2) that the market risk premium under the consensus belief is given by

$$
\mathbb{E}_{a}\left[r_{m}-r_{f}\right]=\frac{1}{\tau_{a}} \sigma_{a}^{2}\left[r_{m}\right]
$$

where $\mathbb{E}_{a}\left[r_{m}\right]=\boldsymbol{\pi}_{m}^{T} \boldsymbol{\mu}_{a}$ and $\sigma_{a}^{2}\left[r_{m}\right]=\boldsymbol{\pi}_{m}^{T} \boldsymbol{\Sigma}_{a} \boldsymbol{\pi}_{m}$ are the expected return and the variance of the market portfolio under the consensus belief.

\footnotetext{
${ }^{5}$ The market clearing condition simply says that the market capitalization of each risky asset must equal to the total wealth invested in the asset. The portfolio weights of the market portfolio $\boldsymbol{\pi}_{m}$ is endogenously determined in our model since it is determined by the heterogeneous beliefs of the agents. If one defines the market portfolio by the total supply of shares of risky assets $\mathbf{z}_{m}$, then the market clearing condition becomes $P_{0}^{-1} \sum_{i=1}^{N} \boldsymbol{\pi}_{i}^{*}=\mathbf{z}_{m}$, where $P_{0}$ is a diagonal matrix of equilibrium prices for risky assets. From this condition, one can compute the equilibrium asset prices.
} 
C. A Benchmark Economy. To examine the impact of heterogeneity, we introduce a benchmark economy in which all agents have the same risk tolerance $\tau$ and homogeneous belief $\mathcal{B}_{o}:=\left(\boldsymbol{\mu}_{o}, \boldsymbol{\Sigma}_{o}\right)$, where $\boldsymbol{\mu}_{o}=\left(\mu_{1}, \mu_{2}, \cdots, \mu_{K}\right)^{T}$ and $\boldsymbol{\Sigma}_{o}=\left(\rho_{j k} \sigma_{j} \sigma_{k}\right)_{K \times K}$ with $\mu_{k}=\mathbb{E}\left[r_{k}\right], \sigma_{k}^{2}=\operatorname{Var}\left[r_{k}\right]$ and $\rho_{j k}=\operatorname{Correl}\left[r_{j}, r_{k}\right]$. The homogeneous belief held by all the agents is referred to as the objective belief, which can be thought of as the hypothetical "true belief" about the expected returns and the covariance matrix. In the benchmark economy, the weights of the market portfolio are given by $\hat{\boldsymbol{\pi}}_{m}=\tau \boldsymbol{\Sigma}_{o}^{-1}\left(\boldsymbol{\mu}_{o}-\hat{r}_{f}\right)$ with the return $\hat{r}_{m}=\hat{\boldsymbol{\pi}}_{m}^{T} \mathbf{r}$. The risk-free rate $\hat{r}_{f}$ is obtained by replacing $\boldsymbol{\Sigma}_{a}$ and $\boldsymbol{\mu}_{a}$ by $\boldsymbol{\Sigma}_{o}$ and $\boldsymbol{\mu}_{o}$ respectively in equation (4) and consequently the market risk premium is given by

$$
\mathbb{E}\left[\hat{r}_{m}-\hat{r}_{f}\right]=\frac{1}{\tau} \sigma^{2}\left[\hat{r}_{m}\right] .
$$

Equation (6) shows that, under the homogeneous belief, the market risk premium is completely characterized by the variance of the market portfolio and the risk tolerance. Since the consensus belief coincides with the objective belief, the market risk premiums under the consensus belief and the objective belief are the same.

\section{The EFFECT OF Dis AGREEMENT}

To examine the effect of heterogeneity in a multi-asset market, we consider an economy with two risky assets and two agents with different risk tolerances and heterogeneous subjective beliefs about the expected returns, standard deviations and the correlation coefficient. We characterize agents' risk tolerances and subjective beliefs as mean preserving spreads (MPS) of the average risk tolerance and the objective belief. We show that the impact of disagreement in general does not cancel out. Moreover, heterogeneity in beliefs and risk tolerances can have very different impact on market equilibrium in compare with a market with a single risky asset.

A. Belief Structure - Mean-Preserving Spreads. To examine whether the effect of heterogeneity cancels out, we assume that on average, the level of risk tolerance and subjective belief in the economy are the same as in the benchmark economy. More precisely, we assume the risk tolerances of the two agents are given by

$$
\tau_{1}=\tau\left(1-\varepsilon_{\tau}\right), \quad \tau_{2}=\tau\left(1+\varepsilon_{\tau}\right) .
$$

The difference in risk tolerances is measured by $\varepsilon_{\tau}$. When $\varepsilon_{\tau}>(<) 0$, agent 1 is less (more) risk tolerant than agent 2. Moreover, for each asset $k=1,2$, the subjective 
beliefs of the two agents are given by

$$
\begin{aligned}
& \mu_{1, k}=\mu_{k}-\varepsilon_{\mu, k} \sigma_{k}, \quad \mu_{2, k}=\mu_{k}+\varepsilon_{\mu, k} \sigma_{k} ; \\
& \sigma_{1, k}=\sigma_{k}\left(1-\varepsilon_{\sigma, k}\right), \quad \sigma_{2, k}=\sigma_{k}\left(1+\varepsilon_{\sigma, k}\right), \\
& \rho_{1}=\rho\left(1-\varepsilon_{\rho}\right), \quad \rho_{2}=\rho\left(1+\varepsilon_{\rho}\right) .
\end{aligned}
$$

In equation (8), $\varepsilon_{\mu, k}, \varepsilon_{\sigma, k}$ and $\varepsilon_{\rho}$ measure the differences in agents' perceived expected returns $\left(\mu_{i, k}\right)$, variances of returns $\left(\sigma_{i, k}\right)$, and correlation coefficients $\left(\rho_{i}\right)$, respectively. When $\varepsilon_{\mu, k}>(<) 0$, agent 1 is relatively pessimistic (optimistic) about the return of asset $k$ than agent 2 ; when $\varepsilon_{\sigma, k}>(<) 0$, agent 1 is relatively confident (doubtful) about the return of asset $k$ than agent 2 ; when $\varepsilon_{\rho}>(<) 0$, agent 1 perceives a lower (higher) correlation between asset returns than agent 2 .

B. Impact on the Consensus Belief. When the consensus belief coincides with the objective belief, the effect of heterogeneity in beliefs and risk tolerances cancels out. However, in the following propositions, we show that the effect of heterogeneity only cancels out in very special cases. In general the heterogeneity can have significant impact on the consensus belief and the impact can be very different in a market with two risky assets in compare with a market with a single risky asset ${ }^{6}$.

Proposition 1. When agents are optimistic/pessimistic about the asset returns and have different risk tolerances ( so that $\varepsilon_{\mu, k}, \varepsilon_{\tau} \in(-1,1)$ and $\varepsilon_{\sigma, k}=\varepsilon_{\rho}=0$ in $(8)$ ), the consensus belief is given by

$$
\Sigma_{a}=\Sigma_{o}, \quad \boldsymbol{\mu}_{a}=\left(\mu_{1}+\theta_{1}, \mu_{2}+\theta_{2}\right)^{T}
$$

where $\theta_{k}=\sigma_{k} \varepsilon_{\mu, k} \varepsilon_{\tau}$ for $k=1,2$.

Proposition 1 shows that, when beliefs are homogeneous about the covariance matrix, the market perceives the objective covariance matrix $\Sigma_{o}$. The market also perceives the objective expected returns $\boldsymbol{\mu}_{o}$ when agents have the same levels of risk tolerance (so that $\epsilon_{\tau}=0$ ). However, when risk tolerances differ, the market becomes over-optimistic (over-pessimistic) if the risk tolerance and optimism (pessimism) are positively correlated meaning that the more risk tolerant agent is more optimistic (pessimistic) than the less risk tolerant agent. Intuitively, the more risk tolerant agent has a stronger incentive to buy/sell the risky assets, therefore his belief about the expected returns has a larger weight in determining the consensus belief.

Proposition 2. When agents are optimistic/pessimistic and confident/doubtful about asset returns (so that $\varepsilon_{\mu, k}, \varepsilon_{\sigma, k} \in(-1,1)$ and $\varepsilon_{\rho}=\varepsilon_{\tau}=0$ in $(8)$ ), the consensus belief

\footnotetext{
${ }^{6}$ The proofs of all the propositions follow from the consensus belief and hence are omitted.
} 
satisfies

$$
\begin{aligned}
& \left(\mu_{1}-\mu_{a, 1}\right) / \sigma_{1}=\omega_{1}\left(\varepsilon_{\sigma, 1} \varepsilon_{\mu, 1}\right)+\omega_{2}\left(\varepsilon_{\sigma, 2} \varepsilon_{\mu, 2}\right), \\
& \left(\mu_{2}-\mu_{a, 2}\right) / \sigma_{2}=\omega_{1}\left(\varepsilon_{\sigma, 2} \varepsilon_{\mu, 2}\right)+\omega_{2}\left(\varepsilon_{\sigma, 1} \varepsilon_{\mu, 1}\right),
\end{aligned}
$$

where

$$
\begin{aligned}
& \omega_{1}=\frac{\left(2-\rho^{2}\right)}{1+\varepsilon_{\sigma, 1}^{2}-\rho^{2}}, \quad \omega_{2}=\frac{\rho}{1+\varepsilon_{\sigma, 2}^{2}-\rho^{2}}, \\
& \sigma_{a, 1}^{2}=\gamma_{1} \sigma_{1}^{2}, \quad \sigma_{a, 2}^{2}=\gamma_{2} \sigma_{2}^{2}, \quad \rho_{a}=\gamma_{\rho} \rho,
\end{aligned}
$$

and

$$
\begin{aligned}
\gamma_{1} & =\frac{\left(1-\varepsilon_{\sigma, 1}^{2}\right)^{2}\left(1+\varepsilon_{\sigma, 2}^{2}\right)\left(1-\rho^{2}\right)}{\left(1-\rho^{2}\right)\left(1+\varepsilon_{\sigma, 1} \varepsilon_{\sigma, 2}\right)^{2}+\left(\varepsilon_{\sigma, 1}-\varepsilon_{\sigma, 2}\right)^{2}}, \\
\gamma_{2} & =\frac{\left(1-\varepsilon_{\sigma, 2}^{2}\right)^{2}\left(1+\varepsilon_{\sigma, 1}^{2}\right)\left(1-\rho^{2}\right)}{\left(1-\rho^{2}\right)\left(1+\varepsilon_{\sigma, 1} \varepsilon_{\sigma, 2}\right)^{2}+\left(\varepsilon_{\sigma, 1}-\varepsilon_{\sigma, 2}\right)^{2}}, \\
\gamma_{\rho} & =\frac{1+\varepsilon_{\sigma, 1} \varepsilon_{\sigma, 2}}{\sqrt{\left(1+\varepsilon_{\sigma, 1}^{2}\right)\left(1+\varepsilon_{\sigma, 2}^{2}\right)}} .
\end{aligned}
$$

Proposition 2 shows that the difference between the consensus and objective beliefs about the expected return of each asset is a weighted average of the covariance between pessimism and confidence measured by $\varepsilon_{\sigma, k} \varepsilon_{\mu, k}$. When pessimism and confidence are positive (negatively) correlated, that is $\varepsilon_{\sigma, k} \varepsilon_{\mu, k}>(<) 0$, the market is more likely to be over-pessimistic (optimistic) about the return of asset $k$. This is intuitive, since the confident agent is more willing to trade in the risky asset, therefore the market should reflect more of his subjective belief about the expected asset return. However, different from a market with a single risky asset, equation (10) shows that, for example, even if agents agree on the expected return of asset 1 (so that $\epsilon_{\mu, 1}=0$ ), a positive correlation between pessimism and confidence about the return of asset 2 (so that $\varepsilon_{\sigma, 2} \varepsilon_{\mu, 2}>0$ ) can lead the market to be over-pessimistic for both assets (so that $\left.\mu_{a, k}<\mu_{k}(k=1,2)\right)$ when there is a positive correlation between asset returns $(\rho>0)$, but be over-optimistic for asset 1 and over-pessimistic for asset 2 when there is a negative correlation $(\rho>0)$. Similarly, equation (11) shows that, for example, even if agents agree on the standard deviation of asset 1 (so that $\epsilon_{\sigma, 1}=0$ ), the market can still be over-confident, that is $\sigma_{a, k}<\sigma_{k}(k=1,2)$ when agents perceive different standard deviations of asset 2. Furthermore, agents in general do not perceive the objective correlation coefficient, that is $\rho_{a} \neq \rho$ when agents have different levels of disagreement about the standard deviations of assets 1 and 2 . Note that $\rho_{a}=\rho$ only when $\varepsilon_{\sigma, 1}=\varepsilon_{\sigma, 2}$. This type of spill-over effect, the fact that disagreement about the expected return and the standard deviation of one asset can also affect the consensus belief about the entire joint distribution of both assets, disappears when the asset returns are uncorrelated. 
Intuitively, when $\rho=0$, agent $i$ 's demand for asset 1 only depends on his perceived expected return and the variance of asset 1 , that is $\pi_{i, 1}=\tau\left(\mu_{i, 1}-r_{f}\right) / \sigma_{i, 1}^{2}$. Therefore, when both agents perceive the objective expected return and variance for asset 1 , so does the market under the consensus belief. However, when $\rho \neq 0$, due to the hedging demand, agent $i$ 's demand for asset 1 also depends on his perceived expected return and standard deviation of asset 2 , that is

$$
\pi_{i, 1}=\frac{\tau\left(\mu_{i, 1}-r_{f}\right)}{\sigma_{i, 1}^{2}}-\underbrace{\frac{\tau \rho}{1-\rho^{2}}\left[\rho \frac{\left.\mu_{i, 1}-r_{f}\right)}{\sigma_{i, 1}^{2}}-\frac{\left.\mu_{i, 2}-r_{f}\right)}{\sigma_{i, 1} \sigma_{i, 2}}\right]}_{\text {hedging demand }} .
$$

Therefore the spill-over effect is due to the hedging demand 7

Proposition 3. When agents are optimistic/pessimistic about asset returns and have different beliefs about the correlation coefficient (so that $\varepsilon_{\mu, k}, \varepsilon_{\rho} \in(-1,1)$ and $\varepsilon_{\sigma, k}=$ $\varepsilon_{\tau}=0$ in (8)), the consensus belief satisfies

$$
\frac{\mu_{a, 1}-\mu_{1}}{\sigma_{1}}=\frac{\rho^{2} \varepsilon_{\rho}}{1-\rho^{2}}\left[\varepsilon_{\mu, 1}-\frac{\varepsilon_{\mu, 2}}{\rho}\right], \quad \frac{\mu_{a, 2}-\mu_{2}}{\sigma_{2}}=\frac{\rho^{2} \varepsilon_{\rho}}{1-\rho^{2}}\left[\varepsilon_{\mu, 2}-\frac{\varepsilon_{\mu, 1}}{\rho}\right],
$$

and

$$
\sigma_{a, 1}^{2}=\sigma_{1}^{2}\left[1-\frac{\rho^{2} \varepsilon_{\rho}^{2}}{1-\rho^{2}}\right], \quad \sigma_{a, 2}^{2}=\sigma_{2}^{2}\left[1-\frac{\rho^{2} \varepsilon_{\rho}^{2}}{1-\rho^{2}}\right], \quad \rho_{a}=\left[\frac{1-\rho^{2}\left(1-\varepsilon_{\rho}^{2}\right)}{1-\rho^{2}\left(1+\varepsilon_{\rho}^{2}\right)}\right] \rho .
$$

Proposition 3 shows the joint impact of the optimism/pessimism and the dispersion in beliefs about the correlation coefficient. Equation (12) shows that a positive correlation between the optimism and the perceived correlation coefficient for asset 1 (such that $\varepsilon_{\rho} \varepsilon_{\mu, 1}>0$ ) leads over-optimism for the return of asset 1 and over-pessimism for the return of asset 2. There is also a similar spill-over effect when $\varepsilon_{\rho} \varepsilon_{\mu, 2}>0$. In equation (13), the difference in the perceived correlation coefficients leads to an overconfidence in the consensus belief $\left(\sigma_{a, k}<\sigma_{k}\right.$ for $\left.k=1,2\right)$ and a higher correlation coefficient $\left(\rho_{a}>\rho\right)$. Intuitively, in this case, the spill-over effect is due to the hedging demand of the agent who perceives a higher correlation between asset returns since he would trade more aggressively than the agent who perceives a lower correlation coefficient. The spill-over disappears when $\rho=0$ since there is no more hedging demand.

In summary, when the heterogeneity in agents' beliefs and the risk tolerances are MPS of the benchmark belief, there can be significant differences between the consensus and the objective beliefs. In particular, we find that when the optimism/pessimism is correlated with the confidence/doubt or the perceived correlation coefficients, there is a spill-over effect, that is, the disagreements about the expected return and standard

\footnotetext{
${ }^{7}$ We would like to thank the referee for pointing this out.
} 
deviation of one asset affect the consensus belief about the entire joint distribution of both assets. The spill-over effect is due to the hedging demand of the over-confident agent or the agent who perceives a higher correlation between the asset returns.

\section{Impact on the Market Risk Premium and the Risk-Free Rate}

Following Abel (2002), we differentiate between two measures of the market risk premium. The first measure $\mathbb{E}\left[r_{m}-r_{f}\right]$ is the expected risk premium of the market portfolio under the objective belief. The second measure $\mathbb{E}_{a}\left[r_{m}-r_{f}\right]$ is the expected risk premium perceived under the consensus belief. We refer to the first measure as the objective risk premium and the second measure as the consensus risk premium respectively. These two measures are generally different. To illustrate the difference between the two measures, we introduce the following decompositions,

$$
\begin{aligned}
& \mathbb{E}\left[r_{m}-r_{f}\right]=\mathbb{E}\left[\hat{r}_{m}-\hat{r}_{f}\right]+P+\frac{1}{\tau_{a}} D+\frac{1}{\tau_{a}} \Delta V, \\
& \mathbb{E}_{a}\left[r_{m}-r_{f}\right]=\mathbb{E}\left[\hat{r}_{m}-\hat{r}_{f}\right]+\frac{1}{\tau_{a}} D+\frac{1}{\tau_{a}} \Delta V, \\
& r_{f}=\hat{r}_{f}-P-\frac{1}{\tau_{a}} D-\frac{1}{\tau_{a}} \Delta V+\Delta R .
\end{aligned}
$$

In equations (14) - (16), $P=\mathbb{E}\left[r_{m}\right]-\mathbb{E}_{a}\left[r_{m}\right]$ and $D=\sigma_{a}^{2}\left[r_{m}\right]-\sigma^{2}\left[r_{m}\right]$ measure the amount of pessimism and doubt in the consensus belief about the return of the market portfolio. The terms $\Delta V=\sigma^{2}\left[r_{m}\right]-\sigma^{2}\left[\hat{r}_{m}\right]$ and $\Delta R=\mathbb{E}\left[r_{m}\right]-\mathbb{E}\left[\hat{r}_{m}\right]$ measure the increase in the variance and expected return of the market portfolio under the objective belief due to the changes in market portfolio weights. Equation (14) shows that the objective risk premium increases with pessimism and doubt in the consensus belief, and also increases with market variance; while equation (15) shows that the consensus risk premium is not affected by pessimism. Intuitively, when the market is pessimistic about the asset returns, there is a greater demand for the risk-free asset, which reduces the risk-free rate and increases the market risk premium. However, it also means that the market expects a lower return from the market portfolio, which cancels out with the positive effect from the reduction of the risk-free rate. Therefore, although pessimism increases the objective risk premium, it does not affect the consensus risk premium. This result is also obtained in Abel (2002). Equation (16) shows that the risk-free rate decreases with the pessimism and doubt in the consensus belief, it also decreases with the market variance, but increases with the expected market return. When the market portfolio under the consensus belief is the same as the one under the objective belief, that is $\boldsymbol{\pi}_{m}=\hat{\boldsymbol{\pi}}_{m}$, the changes in the market variance $\Delta V$ and the expected return $\Delta R$ are both zero. In particular, this would be the case for the economy with single risky asset, in which $\pi_{m}=\hat{\pi}_{m}=1$. In this case, the objective risk premium 
$\mathbb{E}\left[r_{m}-r_{f}\right]$ increases and the risk-free rate decreases when the market is pessimistic and doubtful, as in Abel (2002) and Jouini and Napp (2006). In addition, consistent with Abel (2002), the consensus risk premium $\mathbb{E}_{a}\left[r_{m}-r_{f}\right]$ increases only with doubt, but not pessimism.

To understand the difference in the impact of heterogeneity on the market risk premium and the risk-free rate between a market with two risky assets and a market with a single risky asset, we consider two different scenarios. One is when the market portfolio remains the same as in the benchmark economy, and the other scenario is when the market portfolio is different from the one under the benchmark economy,a more general case. When the agents with different risk tolerances are optimismist/pessismistic, we are able to analyze the changes in the market risk premium and the risk-free rate explicitly in the following proposition. For other cases when the risk tolerance, opti$\mathrm{mism} /$ pessism and dobut/confidence are correlated, due to complexity, the impact of heterogeneity is analyzed through numerical examples.

Proposition 4. When agents with different risk tolerances are optimistic/pessimistic about asset returns (so that $\varepsilon_{\mu, k}, \varepsilon_{\tau} \in(-1,1)$ and $\varepsilon_{\sigma, k}=\varepsilon_{\rho}=0$ in (8)),

(i) the change in the weights of the market portfolio is given by

$$
\boldsymbol{\pi}_{m}-\hat{\boldsymbol{\pi}}_{m}=\frac{\tau}{\sigma_{1}^{2}-2 \rho \sigma_{1} \sigma_{2}+\sigma_{2}^{2}}\left(\theta_{1}-\theta_{2}, \theta_{2}-\theta_{1}\right)^{T}
$$

(ii) the change in the risk-free rate is given by

$$
r_{f}-\hat{r}_{f}=\frac{\theta_{1}\left(\sigma_{2}^{2}-\rho \sigma_{1} \sigma_{2}\right)+\theta_{2}\left(\sigma_{1}^{2}-\rho \sigma_{1} \sigma_{2}\right)}{\sigma_{1}^{2}-2 \rho \sigma_{1} \sigma_{2}+\sigma_{2}^{2}} ;
$$

(iii) the change in the objective risk premium is given by

$$
\mathbb{E}\left[r_{m}-r_{f}\right]-\mathbb{E}\left[\hat{r}_{m}-\hat{r}_{f}\right]=\left(\hat{r}_{f}-r_{f}\right)+\frac{\tau\left(\theta_{1}-\theta_{2}\right)\left(\mu_{1}-\mu_{2}\right)}{\sigma_{1}^{2}-2 \rho \sigma_{1} \sigma_{2}+\sigma_{2}^{2}} ;
$$

and the change in the consensus risk premium is given by

$$
\mathbb{E}_{a}\left[r_{m}-r_{f}\right]-\mathbb{E}\left[\hat{r}_{m}-\hat{r}_{f}\right]=\frac{\tau\left(\theta_{1}-\theta_{2}\right)\left(\theta_{1}-\theta_{2}+2\left(\mu_{1}-\mu_{2}\right)\right)}{\sigma_{1}^{2}-2 \rho \sigma_{1} \sigma_{2}+\sigma_{2}^{2}} .
$$

Parameters $\theta_{1}$ and $\theta_{2}$ defined in Proposition 1 can be used to measure the covariances between the risk tolerance and the optimism/pessimism. When the covariances are the same so that $\theta_{1}=\theta_{2}=\theta$, equation (17) shows that the market portfolio remains the same as in the benchmark economy. In general, the market portfolio changes and the pessimism and doubt are no longer necessary to characterize the changes in the market risk premium and the risk-free rate.

A. Effect of Pessimism and Doubt. We first consider the case where the market portfolio remains the same as in the benchmark economy. In this scenario, the market 
expected return and variance under the objective belief are the same as in the benchmark economy. Therefore, the only way that the market risk premium and the risk-free rate can be affected by the heterogeneity in beliefs and risk tolerances is through the pessimism and doubt. More precisely, the objective risk premium, $\mathbb{E}\left[r_{m}-f_{f}\right]$ increases and the risk-free rate decreases when the consensus belief about the return of the market portfolio is pessimistic and doubtful. The consensus risk premium $\mathbb{E}_{a}\left[r_{m}-r_{f}\right]$ only increases with doubt.

When the risk tolerance and the optimism/pessimism are correlated and $\theta_{1}=\theta_{2}=$ $\theta$, Proposition 4 shows that the optimism/pessimism has no effect on the market portfolio. Intuitively, the consensus belief has the same amount of optimism/pessimism about the return of each asset. Hence, as indicated by Proposition 1, the market portfolio does not change. Also, we have from equations (18) and (19) that

$$
\mathbb{E}\left[r_{m}-r_{f}\right]-\mathbb{E}\left[\hat{r}_{m}-\hat{r}_{f}\right]=-\theta, \quad r_{f}-\hat{r}_{f}=\theta .
$$

This result and its intuition are consistent with Jouini and Napp (2006) for a market with a single risky asset. That is, a positive correlation between risk tolerance and pessimism (so that $\theta<0$ ) makes the market over-pessimistic, leading to an increase in the market risk premium and a decrease in the risk-free rate. Since there is no change in the market portfolio, there is no change in the market variance and expected return. Hence both $\Delta V$ and $\Delta R$ are equal to zero in equations (14) and (16). Therefore, only the pessimism $P=-\theta$ affects the market risk premium and the risk-free rate $]^{8}$ Furthermore, under the consensus belief, the reduction in the risk-free rate is cancelled out by the fact that the market expects lower returns for risky assets. Hence the consensus risk premium is always the same as in the benchmark economy $\mathbb{E}_{a}\left[r_{m}-r_{f}\right]=\mathbb{E}\left[\hat{r}_{m}-\hat{r}_{f}\right]$.

To illustrate the impact, we conduct some numerical examples. As a benchmark, let the two risky assets in the economy have expected returns $\left(\mu_{1}, \mu_{2}\right)=(0.06,0.09)$, standard deviations $\left(\sigma_{1}, \sigma_{2}\right)=(0.08,0.3)$, and correlation coefficient $\rho=0.8$. Both agents hold the objective belief about asset returns, that is, $\mathcal{B}_{i}=\mathcal{B}_{o}=(\boldsymbol{\Sigma}, \boldsymbol{\mu})$ for $i=$ 1,2 . Agents have the same level of risk tolerance given by $\tau=0.5$. From equations (2) and (6), the market portfolio is given by $\hat{\boldsymbol{\pi}}_{m}=(0.962,0.038)^{T}$, the risk-free rate and the market risk premium are given by $\hat{r}_{f}=4.62 \%$ and $\hat{\mathbb{E}}\left(r_{m}-r_{f}\right)=1.49 \%$ respectively, see Tab. 11 Case BM.

For Cases 1 and 2 in Tab. 1, we introduces heterogeneity in beliefs and risk tolerance proportionally for each risky asset such that their weights in the market portfolio remain the same as in the benchmark economy. In Case 1, the more risk tolerant agent

\footnotetext{
${ }^{8}$ The pessimism $P$ measures the average pessimism across assets weighted by their weights in the market portfolio. However, since the amount of optimism/pessimism is the same for each asset, $\theta_{1}=$ $\theta_{2}=\theta$, the average pessimism is simply given by $-\theta$.
} 


\begin{tabular}{|c|c|c|c|}
\hline & BM & Case 1 & Case 2 \\
\hline $\mathbb{E}\left[r_{m}-r_{f}\right]$ & $1.49 \%$ & $3.49 \%$ & $2.87 \%$ \\
\hline $\mathbb{E}_{a}\left[r_{m}-r_{f}\right]$ & $1.49 \%$ & $1.49 \%$ & $1.32 \%$ \\
\hline$r_{f}$ & $4.62 \%$ & $2.62 \%$ & $3.24 \%$ \\
\hline$P$ & $0.00 \%$ & $2.00 \%$ & $1.55 \%$ \\
\hline$D$ & $0.00 \%$ & $0.00 \%$ & $-0.08 \%$ \\
\hline$\pi_{m, 2}$ & 0.038 & 0.038 & 0.038 \\
\hline$\Delta V$ & $0.00 \%$ & $0.00 \%$ & $0.00 \%$ \\
\hline$\Delta R$ & $0.00 \%$ & $0.00 \%$ & $0.00 \%$ \\
\hline
\end{tabular}

TABLE 1. The effects of heterogeneity on the objective risk premium $\mathbb{E}\left[r_{m}-\right.$ $\left.r_{f}\right]$, consensus risk premium $\mathbb{E}_{a}\left[r_{m}-r_{f}\right]$, risk-free rate $r_{f}$, pessimism premium $P$, doubt premium $D$, market portfolio $\boldsymbol{\pi}_{m}$, changes in market variance $\Delta V$ and expected market return $\Delta R$. .

is relatively more pessimistic than the less risk tolerant agent. More precisely, we set $\varepsilon_{\tau}=-0.5, \varepsilon_{\mu, 1}=0.5$ and $\varepsilon_{\mu, 2}=0.1333$. As a result, the amount of pessimism is the same for each asset, that is $P=\theta_{1}=\theta_{2}=0.02$. Results in Tab. 11 Case 1 show that consistent with the analytical results in Proposition 4, the objective risk premium increases and the risk-free rate decreases by the same amount of the pessimism $P=2 \%$, however the consensus risk premium is unchanged. In Case 2 , the confident agent is relatively more pessimistic than the doubtful agent. We set the parameters $\varepsilon_{\sigma, k}=0.2$ for $k=1,2$ and $\varepsilon_{\mu, 1}=0.5$ and $\varepsilon_{\mu, 2}=0.1629$ such that the market portfolio remains unchanged. Results in Tab. 11 Case 2 show that a positive correlation between confidence and pessimism leads to over-pessimism in the consensus belief ( $P=1.55 \%$ ), which has a positive effect on the objective risk premium. However, dispersion in the perceived standard deviations of returns leads to over-confidence in the consensus belief ( $D=-0.08 \%$ ), reducing both the objective and consensus risk premia. As a result, the objective risk premium only increases by $1.38 \%$, which is less compared to Case 1, and the consensus risk premium is even lower than the benchmark risk premium. The risk-free rate is also reduced by $1.38 \%$, which is consistent with the decomposition in equations (14) and (16). We have also considered the case in which the more risk tolerant agent is relatively more doubtful than the less risk tolerant agent. When the market portfolio remains the same as in the benchmark economy, we find that a positive correlation between the risk tolerance and the doubt leads to a positive doubt premium in both the objective and consensus risk premia, which is consistent with Jouini and Napp (2006). However, the increase in the market risk premium is very small compared to the two cases analyzed in Tab. 1.

In summary, when the heterogeneity in beliefs and risk tolerances does not affect the market portfolio, the two risky assets can be considered as a whole through the market 
portfolio. Then the dispersion in the beliefs and the difference in the risk tolerances affect the market risk premium and the risk-free rate only through pessimism and doubt in the consensus belief. Both theoretical and numerical results show consistency with the intuitions from a market with a single risky asset. Numerically, we show that a positive correlation between the risk tolerance and the pessimism is the most promising in generating a high objective risk premium and a lower risk-free rate compared to the benchmark economy. However, the consensus risk premium is always the same as the benchmark risk premium.

B. Effect of Changes in the Market Portfolio. When the market portfolio under the consensus belief is different from the one under the benchmark economy, pessimism and doubt are no longer necessary to characterize changes in the market risk premium and the risk-free rate. Moreover, some of the results and intuitions for the market with one risky asset may not necessarily carry over to a market with two risky assets. For example, in Case 1, it follows from Proposition 4 that, when $\theta_{1} \neq \theta_{2}$, it is not necessarily true that only a positive correlation between the risk tolerance and pessimism can increase the objective risk premium and reduce the risk-free rate. In fact, we observe from equations (18) and (19) that even when risk tolerance and optimism are positively correlated (so that $\theta_{k}>0$ for $k=1,2$ ), the risk-free rate is reduced when $\theta_{1}\left(\sigma_{2}^{2}-\rho \sigma_{1} \sigma_{2}\right)+\theta_{2}\left(\sigma_{1}^{2}-\rho \sigma_{1} \sigma_{2}\right)<1$. For example, if returns are positively correlated and the return of asset 2 is much more volatile than the return of asset 1 , such that $\sigma_{1}<\rho \sigma_{2}$ (which implies $\sigma_{2}>\rho \sigma_{1}$ ), then a positive correlation between the risk tolerance and optimism leads to a reduction in the risk-free rate if $\theta_{1} / \theta_{2}<\sigma_{1}\left(\rho \sigma_{2}-\sigma_{1}\right) /\left(\sigma_{2}\left(\sigma_{2}-\rho \sigma_{1}\right)\right)<1$. Intuitively, when the market is overoptimistic about asset returns, the proportion of asset 2 in the market portfolio increases when $\theta_{1}<\theta_{2}$, leading to a higher market variance since asset 2 is more volatile. An increase in the market variance produces a positive $\Delta V$, which increases the market risk premium and reduces the risk-free rate, following equations (14), (15) and (16).

Furthermore, when assets have the same expected returns $\mu_{1}=\mu_{2}$, equation (19) shows that the increase in the objective risk premium is exactly equal to the reduction in the risk-free rate, that is $\mathbb{E}\left[r_{m}-r_{f}\right]-\mathbb{E}\left[\hat{r}_{m}-\hat{r}_{f}\right]=\hat{r}_{f}-r_{f}$. Intuitively, when the expected returns are the same, the heterogeneity cannot affect the market expected return through its impact on the market portfolio. Thus, from equations (14) and (16), the change in the market expected return is always zero, that is $\Delta R=0$. When $\mu_{1} \neq$ $\mu_{2}$, equation (19) shows that the increase in objective risk premium may not be the same as the reduction in the risk-free rate, the additional term depends on the product $\left(\theta_{1}-\theta_{2}\right)\left(\mu_{1}-\mu_{2}\right)$. When this product is positive (negative), we have $\Delta R>(<) 0$, which implies $\mathbb{E}\left[r_{m}-r_{f}\right]-\mathbb{E}\left[\hat{r}_{m}-\hat{r}_{f}\right]>(<) \hat{r}_{f}-r_{f}$. Intuitively, the proportion 
of asset 2 increases in the market portfolio when $\theta_{1}<\theta_{2}$. If asset 2 has a relatively higher (lower) expected return $\mu_{2}>(<) \mu_{1}$, then the expected market return increases (decreases), meaning $\Delta R>(<) 0$.

\begin{tabular}{|c|c|c|c|c|}
\hline & BM & Case 1 & Case 2 & Case 3 \\
\hline $\mathbb{E}\left[r_{m}-r_{f}\right]$ & $1.49 \%$ & $4.49 \%$ & $4.00 \%$ & $6.09 \%$ \\
\hline $\mathbb{E}_{a}\left[r_{m}-r_{f}\right]$ & $1.49 \%$ & $9.51 \%$ & $7.60 \%$ & $7.76 \%$ \\
\hline$r_{f}$ & $4.62 \%$ & $3.46 \%$ & $3.81 \%$ & $1.64 \%$ \\
\hline$P$ & $0.00 \%$ & $-5.02 \%$ & $-3.60 \%$ & $-1.67 \%$ \\
\hline$D$ & $0.00 \%$ & $0.00 \%$ & $-0.49 \%$ & $-0.15 \%$ \\
\hline$\pi_{m, 2}$ & 0.038 & 0.65 & 0.602 & 0.575 \\
\hline$\Delta V$ & $0.00 \%$ & $4.01 \%$ & $3.54 \%$ & $3.29 \%$ \\
\hline$\Delta R$ & $0.00 \%$ & $1.84 \%$ & $1.69 \%$ & $1.62 \%$ \\
\hline
\end{tabular}

TABLE 2. The effects of heterogeneity on the objective risk premium $\mathbb{E}\left[r_{m}-\right.$ $\left.r_{f}\right]$, consensus risk premium $\mathbb{E}_{a}\left[r_{m}-r_{f}\right]$, risk-free rate $r_{f}$, pessimism premium $P$, doubt premium $D$, the market portfolio $\pi_{m}$, changes in market variance $\Delta V$ and expected market return $\Delta R$.

To examine the impact of heterogeneity in beliefs and risk tolerance on the market equilibrium, we consider three cases and compare the market risk premium and the risk-free rate with those under the benchmark economy (Case BM). Numerical results are provided in Tab. 2. In Case 1, we assume a positive correlation between risk tolerance and optimism (so that $\varepsilon_{\tau} \varepsilon_{\mu, k}>0$ for $k=1,2$ ). More specifically, we set $\varepsilon_{\tau}=0.5, \varepsilon_{\mu, 1}=0.1$ and $\varepsilon_{\mu, 2}=0.5$ respectively such that $\theta_{1}<\theta_{2}$. Results in Case 1 of Tab. 2 show that the objective risk premium increases by $3.00 \%$ while the risk-free rate is reduced by $1.16 \%$. The increase in the market variance contributes $\Delta V / \tau_{a}=8.02 \%$ to $\mathbb{E}\left[r_{m}-r_{f}\right]-\mathbb{E}\left[\hat{r}_{m}-\hat{r}_{f}\right]$ and $\hat{r}_{f}-r_{f}$, which is offseted by a negative pessimism premium of $P=-5.02 \%$ due to market's over-optimism. Also, consistent with the analytical result in Proposition 4, the increase in the objective risk premium is larger than the reduction in risk-free rate. In Case 2, we assume that there is a positive correlation between the confidence and optimism. More precisely, we assume $\varepsilon_{\sigma, k}=-0.2$ for $k=1,2, \varepsilon_{\mu, 1}=0.1$ and $\varepsilon_{\mu, 2}=0.5$ such that the market is overoptimistic about asset returns and the over-optimism is stronger for asset 2 than for asset $1\left(-\varepsilon_{\sigma, 1} \varepsilon_{\mu, 1}<-\varepsilon_{\sigma, 2} \varepsilon_{\mu, 2}\right)$. In this case, the market variance increases $(\Delta V>0)$ due to the increase in market's holding of asset 2, which contributes $\Delta V / \tau_{a}=7.08 \%$ to $\mathbb{E}\left[r_{m}-r_{f}\right]-\mathbb{E}\left[\hat{r}_{m}-\hat{r}_{f}\right]$ and $\hat{r}_{f}-r_{f}$. However, this is offseted by the negative pessimism premium of $P=-3.60 \%$ and the negative doubt premium of $D / \tau_{a}=$ $-0.98 \%$ due to the over-optimism and over-confidence in the consensus belief. As a result, the objective risk premium increases by $2.50 \%$ while the risk-free rate reduces by only $0.81 \%$. 
In Case 3, we assume a positive correlation between the optimism and the perceived correlation coefficients. In other words, the optimistic agent perceives a higher correlation between asset returns than the pessimistic agent. More precisely, we set $\varepsilon_{\mu, 1}=0.1, \varepsilon_{\mu, 2}=0.5$ and $\varepsilon_{\rho}=0.2$. This leads to an increase in the market variance and contributes $\frac{1}{\tau_{a}} \Delta V=6.58 \%$ to $\mathbb{E}\left[r_{m}-r_{f}\right]-\mathbb{E}\left[\hat{r}_{m}-\hat{r}_{f}\right]$ and $\hat{r}_{f}-r_{f}$. The negative pessimism premium $(P=-1.67 \%)$ is much smaller compare to Cases 1 and 2, and the negative doubt premium $\left(D / \tau_{a}=-0.30 \%\right)$ is less than that in Case 2. As a result, among all the cases considered, Case 3 provides the highest objective risk premium $\left(\mathbb{E}\left[r_{m}-r_{f}\right]=6.09 \%\right)$ and the lowest risk-free rate $\left(r_{f}=1.64 \%\right)$. Intuitively, from Proposition 3, a positive correlation between the optimism and the perceived correlation coefficients for asset $1\left(\varepsilon_{\rho} \varepsilon_{\mu, 1}>0\right)$ leads to over-optimism about return of asset 1 and over-pessimism about the return of asset 2 in the consensus belief (assume $\rho>0$ ). Therefore the amount of over-optimism in asset 1 is offseted by the amount of overpessimism in asset 2 . The same argument applies when there is a positive correlation between the optimism and the perceived correlation coefficients for asset 2 .

Tab. 2 shows that in all the cases considered, the consensus risk premium is higher than the objective risk premium, that is $\mathbb{E}_{a}\left[r_{m}-r_{f}\right]>\mathbb{E}\left[r_{m}-r_{f}\right]$. This is because the pessimism and doubt premia are either negative or zero. This suggests that in a market with two risky assets, there can be a significant increase in the market risk premium and reduction in the risk-free rate even when the market is over-optimistic and overconfident (instead of being over-pessimistic and doubtful as suggested by Abel (2002) and Jouini and Napp (2006)) about asset returns.

In summary, when the market portfolio is affected by the heterogeneity in beliefs and risk tolerances and different from the one under the benchmark economy, the two assets can no longer be considered as whole through the market portfolio. Consequently, the intuitions from a market with a single risky asset about the way heterogeneity impacts on the market risk premium and the risk-free rate may not carry over to a market with two risky assets. In particular, we have shown that even when the market is overoptimistic and over-confident, there can be a significant increase in the market risk premium and reduction in the risk-free rate.

\section{CONCLusion}

Heterogeneity is both ubiquitous and difficult to model in financial markets. In this paper, we have examined the impact of disagreements among agents in a market with two risky assets and a risk-free asset. When the subjective beliefs and risk tolerance of the agents are characterized by MPS of a benchmark objective belief and the average risk tolerance, we show that the impact of disagreements on the market with two risky 
assets can be very different from that with a single risky asset. First, there is a spill-over effect in the impact of disagreement on the consensus belief, that is disagreement about expected return and standard deviation of one asset can affect the joint distribution of both asset in market equilibrium. Therefore, in a multi-asset market, the consensus belief is generally different from the objective belief and effects of heterogeneity in beliefs and risk tolerance do not cancel out in equilibrium.

We also analyze the impact of disagreement on the market risk premium and the risk-free rate. We show that the intuitions from a market with a single risky asset carry over to the market with two risky assets only when the market portfolio is not affected by the heterogeneity among the agents. In this case the two assets can be considered as whole through the market portfolio. However, in general, the heterogeneity in beliefs and risk tolerances does affect the market portfolio. In this case, the intuitions from a market with a single risky asset may no longer carry over to the market with two risky assets. We find that positive correlations between risk tolerance and optimism or confidence, between confidence and optimism, and between optimism and perceived correlation coefficients can increase the market risk premium and reduce the risk-free rate. The positive correlation between the optimism and the perceived correlation coefficients generates the most significant increase in the market risk premium and reduction in the risk-free rate.

\section{REFERENCES}

Abel, A. (2002), 'An exploration of the effects of pessimism and doubt on asset returns', Journal of Economic Dynamics and Control 26, 1075-1092.

Basak, S. (2000), 'A model of dynamic equilibrium asset pricing with heterogeneous beliefs and extraneous beliefs', Journal of Economic Dynamics and Control 24, 63-95.

Basak, S. (2005), 'Asset pricing with heterogeneous beliefs', Journal of Banking and Finance 29, 28492881.

Berraday, T. (2009), 'Bounded rationality and asset pricing with intermediate consumption', Review of Finance 13, 693-725.

Blume, L. and Easley, D. (2006), 'If you are so smart, why aren't you rich? belief selection in complete and incomplete markets', Econometrica 74, 929-966.

Buraschi, A. and Jiltsov, A. (2006), 'Model uncertianty and option markets with heterogeneous beliefs', Journal of Finance 61, 2814-2897.

Cao, H. and Ou-Yang, H. (2009), 'Differences of opinion of public information and speculative trading in stocks and options', Review of Financial Studies 22, 299-335.

Chiarella, C., Dieci, R. and He, X. (2010), A framework for CAPM with heterogeneous beliefs. in Bishi, G.I., C. Chiarella. and L. Gardini (Eds), Nonlinear Dyanmaics in Economics, Finance and Social Sciences: Essays in Honour of John Barkley Rosser Jr, pp. 353-369, Springer.

Chiarella, C., Dieci, R. and He, X. (2011), 'Do heterogeneous beliefs diversify market risk?', European Journal of Finance 17, 241-258.

David, A. (2008), 'Heterogeneous beliefs, speculation, and the equity premium', Journal of Finance 63, 41-83.

Detemple, J. and Murthy, S. (1994), 'Intertemporal asset pricing with heterogeneous beliefs', Journal of Economic Theory 62, 294-320. 
Duchin, R. and Levy, M. (2010), 'Disagreement, portfolio optimization and excess volatility', Journal of Financial and Quantitative Analysis 45, 623-640.

Dumas, B., Kurshev, A. and Uppal, R. (2009), 'Equilibrium portfolio strategies in the presence of sentiment risk and excess volatility', Journal of Finance 64, 195-229.

Fama, E. and French, K. (2007), 'Disagreement, tastes, and asset prices', Journal of Financial Economics 83, 667-689.

Giordani, P. and Söderlind, P. (2006), 'Is there evidence of pessimism and doubt in subjective distribution? implications for the equity premium puzzle', Journal of Economic Dynamics and Control 30, 1027-1043.

Hong, H. and Stein, J. (2007), 'Disagreement and the stock market', Journal of Economic Prospectives 21, 109-128.

Jouini, E. and Napp, C. (2006), 'Heterogeneous beliefs and asset pricing in discrete time: An analysis of pessimism and doubt', Journal of Economic Dynamics and Control 30, 1233-1260.

Jouini, E. and Napp, C. (2007), 'Consensus consumer and intertemporal asset pricing with heterogeneous beliefs', Review of Economic Studies 74, 1149-1174.

Jouini, E. and Napp, C. (2011), 'Unbiased disagreement in financial markets, waves of pessimism and the risk-return trade-off', Review of Finance 15, 575-601.

Kogan, L., Ross, S., Wang, J. and Westerfield, M. (2006), 'The price impact and survival of irrational traders', Journal of Finance 61, 195-229.

Li, T. (2007), 'Heterogeneous beliefs, asset prices, and volatility in a pure exchange economy', Journal of Economic Dynamics and Control 31, 1697-1727.

Lintner, J. (1969), 'The aggregation of investor's diverse judgements and preferences in purely competitive security markets', Journal of Financial and Quantitative Analysis 4, 347-400.

Mehra, R. and Prescott, E. (1985), 'The equity premium: a puzzle', Journal of Monetary Economics 15, 145-161.

Rubinstein, M. (1974), 'An aggregation theorem for securities markets', Journal of Financial Economics 1, 225-244.

Rubinstein, M. (1975), 'Security market efficiency in an arrow-debreu economy', American Economic Review 65, 812-824.

Weil, P. (1989), 'The equity premium puzzle and the risk-free rate puzzle', Journal of Monetary Economics 24, 401-421.

Xiong, W. and Yan, H. (2010), 'Heterogeneous expectations and bond markets', Review of Financial Studies 23, 1433-1466.

Yan, H. (2010), 'Is noise trading cancelled out by aggregation?', Management Science 57, 1047-1059.

Zapatero, F. (1998), 'Effects of financial innovations on market volatility when beliefs are heterogeneous', Journal of Economic Dynamics and Control 22, 597-626. 